\title{
CLINICAL AND PATHOLOGICAL CHARACTERISTICS OF PATIENTS WITH OVEREXPRESSED HER 2 BREAST CANCER IN THE NATIONAL COHORT STUDY - AMAZONA III
}

Alessandra Borba Anton de Souza1', Gustavo Werutsky², Daniela Dornelles Rosa33, Rafaela Gomes², Sergio Simon ${ }^{4}$ ${ }^{1}$ Pontifícia Universidade Católica do Rio Grande do Sul - Porto Alegre (RS), Brazil. ${ }^{2}$ LACOG, Latin American Cooperative Oncology Group Porto Alegre - Porto Alegre (RS), Brazil. ${ }^{3}$ Hospital Moinhos de Vento - Porto Alegre (RS), Brazil.

${ }^{4}$ Hospital Albert Einsten - São Paulo (SP), Brazil.

Introduction: HER2 overexpressed breast cancer (+) is observed at around 15-20\%. The introduction of target therapies in this subtype changed the prognosis of these patients, improving mortality and reducing surgical morbidity when neoadjuvant therapy allows downstaging of breast and axillary surgery. A recent publication with 1,310 samples from Brazilian patients described $20 \%$ of HER-2 + tumors. There are few epidemiological studies evaluating patients in our population. The Amazona III study evaluated the epidemiology of breast cancer in a sample of the Brazilian population including 2,950 patients from 23 Brazilian hospitals, completed in 2018. Objective: To characterize the frequency of positive HER-2 breast cancer in patients in the Amazona III study and its clinical characteristics. Method: Prospective, multicenter cohort study. All female patients with invasive breast cancer over 18 years of age included in the Amazona III Project were considered eligible. Immunohistochemical determination was made according to the pathology laboratory of each center. Results: Of the 2,950 study participants, 643 (21\%) were HER2 +, 71\% from the Unified Health System (SUS) and 28.9\% from the complementary system. Most had grade 2 (52.6\%) or 3 (36.4\%), were post-menopausal (53\%), positive estrogen receptor (71.9\%), and did not undergo neoadjuvant therapy (52\%). As for the number of lymph nodes, $45 \%$ were positive lymph nodes, with cN1 (27\%), cN2 (12\%), and cN3 (5\%). 49\% had positive lymph nodes in SUS and 37\% in the private system. The rate of conservative breast surgery was similar in both groups, being around $46 \%$. Conclusion: The prevalence of HER-2 + in patients included in the Amazona Study III (current epidemiological study of breast cancer in Brazil) is compatible with data from the literature. The prevalence of positive lymph node at diagnosis is high, especially in SUS (49\%), therefore the impact of access to therapies that increase the chance of increasing overall survival, decreasing invasive recurrence, and decreasing surgical morbidity must be evaluated. 